\title{
Q-V CURVE INTERPRETATIONS OF ENERGY MEASURES FOR VOLTAGE SECURITY
}

\author{
Thomas J. Overbye, Member, IEEE \\ Department of Electrical and Computer Engineering \\ University of Illinois at Urbana-Champaign \\ Urbana, IL 61801
}

\begin{abstract}
Energy methods have shown promise as measures for quantifying the vulnerability of power systems to problems of voltage instability and collapse. However, to make such measures more useful as a security assessment tool in an operational environment, it is important to provide physical interpretations of the quantitative measure. This paper will demonstrate that for certain basic load models, the energy based security measure is equal to the area enclosed by a familiar Q-V curve, with a change of scale on the voltage axis. This interpretation has the added benefit of providing easily computed approximations to the maximum real and reactive power loadability both at individual buses and for the system as a whole. Results are demonstrated in detail on the IEEE 118 bus system, with additional tests on a 415 bus sample system.
\end{abstract}

\section{INTRODUCTION}

As transmission systems become more stressed due to increased loads and large inter-utility power transfers, efficient system operation is becoming increasingly threatened due to problems of voltage instability and collapse [1]. The term voltage instability is generally used to describe situations in which a disturbance, an increase in load, or other system change causes bus voltages to vary significantly from their desired operating range in such a way that standard mechanisms of operator intervention or automatic system controls fail to halt this deviation. If bus voltages ultimately fall in a more rapid decline, leading to loss of portions of the network, the term voltage collapse is applied. These voltage related threats to system security are expected to become more severe over the next decade as demand for electric power rises, while economic and environmental concerns limit the construction of new transmission and generation facilities.

The problems of voltage instability and collapse have attracted considerable attention from power systems researchers, with much of the current work summarized in [1], [2], [3] and references therein. With the accumulating experience of the power system community, there is a growing recognition of the various aspects of the problem, combining as is does both slow and fast time scale phenomena, and quasi-static analysis methods with dynamic models. Convincing models of voltage collapse have been proposed that explain the phenomena as a dynamic consequence of a saddle node bifurcation in

93 WM 184-2 PWRS A paper recommended and approved by the IEEE Power System Engineering Committee of the IEEE Power Englneering Soclety for presentation at the IEEE/PES 1993 winter Meeting, Columbus, OH, January 31 - February 5, 1993. Manuscript submitted August 27, 1992; made available for printing November 30, 1992 .

\author{
Ian Dobson, Member, IEEE and Christopher L. DeMarco, Member, IEEE \\ Department of Electrical and Computer Engineering \\ University of Wisconsin-Madison \\ Madison, WI 53706
}

which the system's operating equilibrium disappears [4]. Other works have explained oscillatory instabilities in voltage related controls as a Hopf bifurcation in which a pair of eigenvalues of the system Jacobian cross the imaginary axis with nonzero imaginary part [5]. In this paper, the term vollage collapse will be reserved for a monotonic decay of voltage away from the desired operating point, and the corresponding analysis is restricted to loss of stable equilibrium through a saddle node bifurcation. The system state is assumed to move from a point of relative security to one of increased vulnerability to voltage instability through a quasi-static variation (time scale of tens of minutes to hours) in system parameters such as the aggregate loads.

Several of the schemes for predicting vulnerability to voltage collapse currently used by utilities [6], [7] employ variations on classic P-V or Q-V curve calculations, which identify the variation in critical bus voltage(s) with respect to changes in loading and/or reactive support. The goal of this paper is to interpret energy function based voltage security measures reported in [8], [9] in terms of Q-V curves. Moreover, a simple geometric relation will be established between the energy based security measure and the allowable increase in load plus losses ("load power margin") at critical system buses or areas. Given the relatively low computational cost involved in computing the energy margin, this relation yields a new approximation to available load or transfer margins with lower computational cost than existing energy based methods described in [8], or alternative approaches in [13], [14].

\section{BACKGROUND AND MOTIVATION}

The energy function voltage security measure is most easily related to the types of Q-V curve analyses as reported in [1, pp. 152-157], [6]. For example, Figure 1 is reproduced from [6], showing voltage at a critical bus in a study system as the fictitious reactive supply at that bus is varied. Note that negative values of reactive supply correspond to increasing reactive load.

The utility practice reported in [6] indicates that it is inappropriate to set a single threshold on either the voltage or reactive power axis of Figure 1, noting that: "Depending on network strength, the power transmitted, and the voltage regulating facilities the shape of the curve could range from very shallow to very steep. For the former, criteria based on voltage margin only might be inadequate since that margin could be consumed by loss of a small amount of reactive support. On the other hand, a criteria based on Mvar margin only might be inadequate if the characteristic is steep." As will be shown in the following section, the energy based voltage security measure is related to area enclosed by the Q-V curve shown in Figure 1, with a modification of the voltage axis to the logarithm of voltage. As a result, either a very shallow curve or a very steep curve will yield a relatively small area enclosed, and a small energy margin. As shown in previous works [8], [9], [10], this margin can be computed at a cost less than that of the repeated power flow calculations necessary to produce a curve of the type shown in Figure 1. Moreover, the following sections will demonstrate how a single energy margin calculation can simultaneously provide information about a number of vulnerable buses in the network.

It is useful to establish some terminology relating to Figure 1. The point labeled as the "voltage instability point" represents the power flow solution obtained when the fictitious reactive source is at its most negative possible value. For values of the reactive source above this 0885-8950/94/\$04.00 @ 1993 IEEE 
332

level (equivalently, lower reactive load), the plot shows the voltage component for two power flow solutions. Those with voltage components on the left "leg" of the curve will here be referred to as "low voltage power flow solutions;" those on the right "leg" will be termed "high voltage power flow solutions." The "voltage instability point" corresponds to the loading level at which these two solutions coalesce into one. If a simple dynamic model is used for a system having only $P-Q$ type loads, then the power flow solutions predict the network related components of its equilibria, and this critical point is a saddle node bifurcation point. In sach a model, the equilibrium associated with the low voltage solution is typically unstable, and will be denoted $x^{4}$, and that associated with the high voltage solution is generally stable, and will be denoted $x^{8}$. It is important to stress, however, that this dynamic interpretation assumes simple load models consistent with the analysis in [6]. While the energy measure can be applied with more general load models (see [8]), the Q-V curve interpretation is strictly appropriate only for the simple $P-Q$ load model.

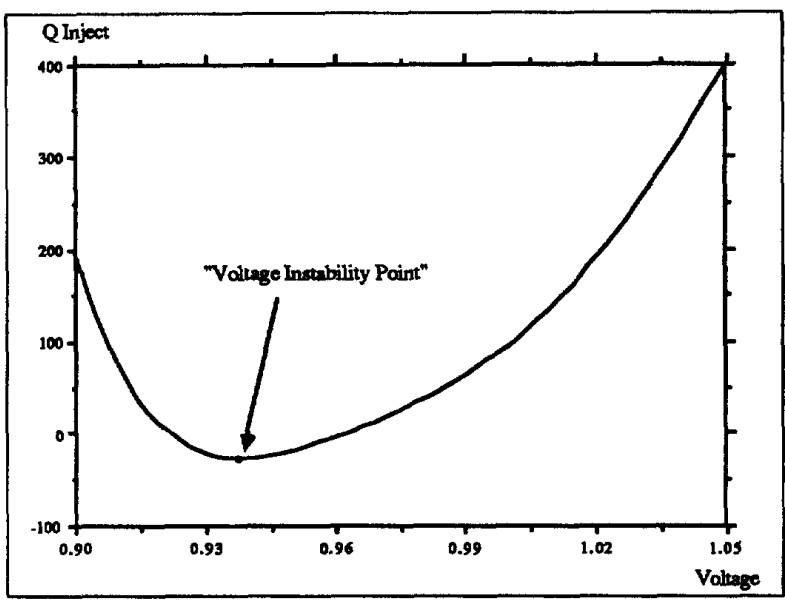

FIGURE 1: Sample Q-V Curve from [6]

\section{ENERGY MEASURE AS AREA UNDER $Q$ vs. $\ln (\mathrm{V})$ CURVE FOR ONE LINE SYSTEM EXAMPLE}

To motivate the relation between the energy based security measure and $Q-V$ curve, we begin by examining a simple one line example with $P-Q$ load demands attached. The parameter change of interest will be an increase in reactive power demand. The following section will extend this analysis to a general $n$-bus network.

Consider a system with a single series transmission line (ignoring shunts) connecting two buses, numbered 1 and 2 . Bus 1 is assumed to be a slack bus, with voltage magnitude fixed at $1.0 \mathrm{pu}$. Transmission losses are also neglected. The load is attached at bus 2 , and is represented as a P-Q demand that is independent of changes in bus voltage magnitude or instantaneous frequency. The parameter change of interest will be a quasi-static increase in the reactive load parameter $Q_{L}$. The resulting power balance equations at bus 2 are:

$$
\begin{aligned}
& f(\alpha, V):=P_{L}+B_{12} V \sin (\alpha)=0 \\
& \bar{g}(\alpha, V):=Q_{L}-B_{22} V^{2}-B_{12} V \cos (\alpha)=0
\end{aligned}
$$

where

$\mathrm{V}:=$ bus voltage magnitude at bus 2

$\alpha:=\delta_{2}-\delta_{1}=$ phase angle difference from bus 2 to bus 1
Observe that for positive bus voltage magnitude, solutions to (1) are unchanged if (1b) is multiplied on both sides by $V^{-1}$, to produce:

$$
g(\alpha, V):=\frac{Q_{L}}{V}-B_{22} V-B_{12} \cos (\alpha)=0
$$

With this modification, the resulting two coupled power balance equations are exactly integrable with respect to $\alpha$ and V. The energy based security measure to indicate vulnerability to voltage collapse is obtained by integrating the function composed of $f(\cdot, \cdot)$ and $g(\cdot, \cdot)$ from a lower limit corresponding to the operating point, denoted $x^{5}=\left(\alpha^{8}, V^{8}\right)$, to an upper limit comesponding to a particular low voltage power flow solution, denoted $x^{u}=\left(\alpha^{u}, V^{u}\right)$. Efficient computational techniques for identifying the appropriate low voltage solution are addressed in [10]. For this simple example, we have:

$$
\begin{aligned}
& x^{\mathrm{u}}=\left(\alpha^{\mathrm{u}}, v^{\mathrm{u}}\right) \\
& \theta\left(x^{u}, x^{s}\right):=\int_{x^{g}=\left(\alpha^{4}, v^{s}\right)}(f(\alpha, V), g(\alpha, V)) \cdot\left[\begin{array}{l}
d \alpha \\
d V
\end{array}\right]= \\
& -\frac{1}{2} B_{22}\left(V^{u}\right)^{2}+\frac{1}{2} B_{22}\left(V^{s}\right)^{2}-B_{12} V^{u} \cos (\alpha u) \\
& +B_{12} V^{s} \cos \left(\alpha^{s}\right)+Q_{L} \ln \left(V^{u} / V^{s}\right)+P_{L}\left(\alpha^{u}-\alpha^{s}\right)
\end{aligned}
$$

The subsequent discussions will make use of the following observations relating to the system model and to (2).

i) We have assumed that the underlying parameter change driving the quasi-static evolution of the operating point is an increase in $\mathrm{Q}_{\mathrm{L}}$. Observe that $Q_{L}$ enters both explicitly in $\theta\left(x^{4}, x^{8}\right)$, and implicitly through the changes in the power flow solutions $x^{5}$ and $x^{4}$ that occur as $\mathrm{Q}_{\mathrm{L}}$ increases. This yields three distinct terms when evaluating the total derivative of $\vartheta\left(x^{4}, x^{5}\right)$ with respect to $Q_{L}$

ii) As established in previous works (see, for examples, [4], [10], and references therein) in this simple model the upper limit of feasible $Q_{L}$ values, denoted $Q_{2}^{\max }$, is given by the loading level where the power flow solutions $x^{5}$ and $x^{4}$ coalesce to a single point. As noted above, this is the "voltage instability point" of Figure 1. It is then clear from the integral definition of $\vartheta\left(x^{4}, x^{3}\right)$ above that the energy measure must have a value of zero at this critical loading level.

iii) Recall the fundamental theorem of calculus. If one differentiates a function with respect to a given variable, and integrates the result with respect to that same variable, one recovers the difference of the function between the upper and lower limits of integration.

(iv) Consider the gradient of $\theta$ with respect to $x^{s}$ or $x^{u}$. From the definition in (2), this yields the power balance equations (1a) and (1b). When evaluated at a power flow solution, these must be zero, so that:

$$
\frac{\partial \vartheta}{\partial x^{s}}\left(x^{s}\left(Q_{L}\right)\right)=\frac{\partial \vartheta}{\partial x^{u}}\left(x^{u}\left(Q_{L}\right)\right)=0
$$

To apply these observations, consider the one line system with a nominal reactive load level of $\mathrm{Q}_{\mathrm{L}}^{0}$, associated operating point $\mathrm{x}^{\mathrm{s}}\left(\mathrm{Q}_{\mathrm{L}}^{0}\right)$, low voltage power flow solution $x^{\mathrm{u}}\left(\mathrm{Q}_{\mathrm{L}}^{0}\right)$, and energy margin $\vartheta\left(x^{\mathrm{u}}\left(Q_{L}^{0}\right), x^{8}\left(Q_{L}^{0}\right)\right)$. If the system load is increased to $Q_{L}^{\max }$, one has $x^{5}\left(Q_{L}^{\max }\right)=x^{4}\left(Q_{L}^{\max }\right)$, and $\theta\left(x^{u}\left(Q_{L}^{\max }\right), x^{8}\left(Q_{L}^{\max }\right)\right)=0$. From (iii) above, it follows that:

$$
\begin{aligned}
& Q_{L}^{\max } \\
& \int_{L}^{0} \frac{d \vartheta}{d Q_{L}} d Q_{L}=0-\vartheta\left(x^{u}\left(Q_{L}^{0}\right), x s\left(Q_{L}^{0}\right)\right)
\end{aligned}
$$

Now consider (i), the form of the three terms that make up $d \vartheta / d Q_{L}$ and their behavior when integrated as in (3) above. One obtains 
$\frac{d \theta}{d Q_{L}}=\frac{\partial \theta}{\partial Q_{L}}+\frac{\partial \theta}{\partial x^{u}} \frac{\partial x^{u}}{\partial Q_{L}}-\frac{\partial \theta}{\partial x^{s}} \frac{\partial x^{*}}{\partial Q_{L}}$

with

$$
\frac{\partial \theta}{\partial Q_{L}}=\ln \left(V^{\mathrm{a}} / N^{3}\right)
$$

The second and third terms of (4) involve the gradient of $\theta$ with respect to $x$; from (iv), it follows that these are zero. Only the first term in (4) contributes to the value of the integral in (3). Therefore:

$$
\begin{aligned}
& \vartheta\left(x^{u}\left(Q_{L}^{0}\right), x^{s}\left(Q_{L}^{0}\right)\right)=-\int_{Q_{L}^{0}}^{Q_{L}^{\max }} \frac{d \vartheta}{d Q_{L}} d Q_{L} \\
& =\int_{Q_{L}^{\max }}^{\int_{L}^{0}}\left[\ln \left(V^{s}\left(Q_{L}\right)\right)-\ln \left(V^{u}\left(Q_{L}\right)\right)\right] d Q_{L}
\end{aligned}
$$

where $V^{s}\left(Q_{L}\right)$ and $V^{u}\left(Q_{L}\right)$ are the voltage magnitude components of $x^{s}\left(Q_{1}\right)$ and $x^{u}\left(Q_{1}\right)$. The right hand side of (5) may be interpreted as the area under a $Q_{L}$ versus $V$ curve, where the voltage magnitude axis is given a natural $\log$ scale. To illustrate this concept, Figure 2 shows the $Q$ vs. $\ln (V)$ curve for the two bus system with a base case loading of $200 \mathrm{MW}$ and $100 \mathrm{Mvar}$. That is, the figure plots the variation in the natural $\log$ of the voltage at bus 2 as a fictitious reactive supply at the bus is varied. The two points where this fictitious reactive injection are zero correspond to the base case power flow solutions for the system (i.e. the normal power flow solution of $V_{2}=0.855$ with natural log of $V_{2}=-0.156$, and the low voltage solution of $V_{2}=0.261$ with natural $\log$ of $V_{2}=-1.343$ ). The energy measure of 0.861 is equal to the shaded per unit area enclosed by the curve between the two power flow solutions. The energy measure thus gives an assessment of system voltage security as the area under the Q-V curve. For this simple load model it will give a quantitative indicator of vulnerability to voltage collapse in a manner similar to the Q-V curve criteria outlined in [6].

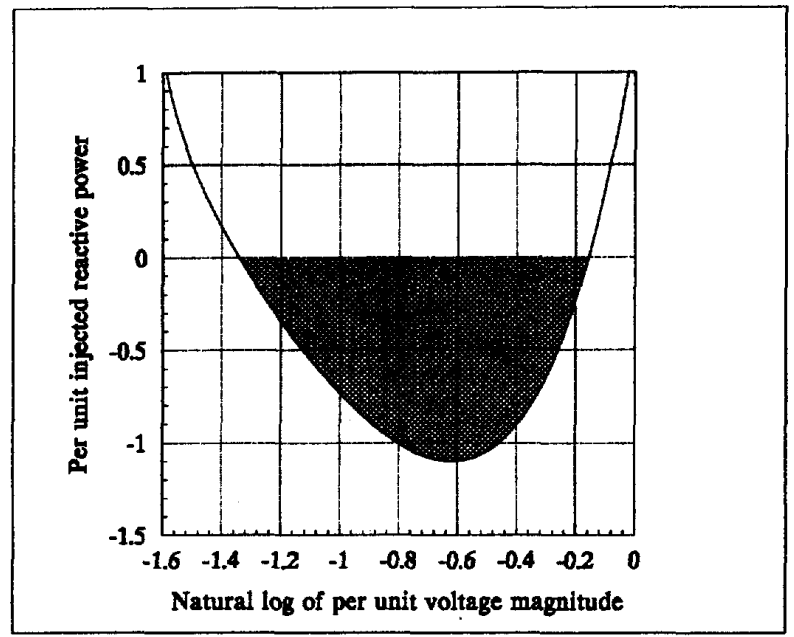

FIGURE 2 : Q- $\ln (\mathrm{V})$ Curve Area Equal to Energy Measure

Work in [8] described how the nearly linear behavior of the voltage security measure could be exploited to give very accurate estimates of the maximum reactive power that can be supplied to a given bus (the reactive margin). However, that method required repeated energy margin calculations over a range of operating points to provide data for a linear extrapolation. The observations here suggest that with only a single energy margin calculation at the current operating point, one can obtain estimates of reactive margin at even lower computational cost. Since the energy measure represents the area under the $Q-\ln (V)$ curve, the reactive margin (i.e. the maximum "depth" of the area) can be approximated by making an assumption about the shape of the area enclosed. Theoretical considerations related to the saddle node bifurcation indicate that this shape is parabolic near the critical point; experience in numerical examples suggests that the area can often be approximated as a parabolic region over a much larger range of load values. Thus the reactive margin, $\mathrm{Q}_{\max }$, at a bus is approximated as

$$
\begin{aligned}
& \text { parabolic area }=\frac{2}{3} \text { base * height } \Rightarrow \\
& \text { energy measure * MVA base } \approx \frac{2}{3} * \ln \left(V^{*}\right)-\ln \left(V^{u}\right) * Q_{\max } \Rightarrow \\
& Q_{\max } \approx \frac{3}{2} \frac{\text { energy measure } * M V A \text { base }}{\ln \left(V^{*}\right)-\ln \left(V^{u}\right)},
\end{aligned}
$$

where the scaling by the MVA base is necessary to convert from the per unit energy measure to the reactive margin in Mvar.

\begin{tabular}{|l|l|l|l|l|l|}
\hline $\begin{array}{l}\text { Mvar load at } \\
\text { bus 2 }\end{array}$ & $\begin{array}{l}\text { Energy } \\
\text { measure }\end{array}$ & \multicolumn{2}{|l|}{$\begin{array}{l}\text { Bus 2 per unil } \\
\text { voltage maqnitude }\end{array}$} & \multicolumn{2}{l|}{$\begin{array}{l}\text { Reactive power } \\
\text { margin (uvar) }\end{array}$} \\
\hline & & $\begin{array}{l}\text { Operable } \\
\text { solution }\end{array}$ & $\begin{array}{l}\text { Low } \\
\text { solution }\end{array}$ & Estimated & Actual \\
\hline 0 & 2.264 & 0.979 & 0.204 & 216.8 & 210.0 \\
\hline 50 & 1.515 & 0.822 & 0.224 & 160.4 & 160.0 \\
\hline 100 & 0.861 & 0.855 & 0.261 & 108.9 & 110.0 \\
\hline 150 & 0.343 & 0.771 & 0.324 & 59.4 & 60.0 \\
\hline 200 & 0.023 & 0.632 & 0.447 & 10.0 & 10.0 \\
\hline 210 & 0.000 & 0.543 & 0.543 & 0.0 & 0.0 \\
\hline
\end{tabular}

TABLE 1 : Estimated vs. Actual Reactive Margin

The accuracy of this estimated reactive margin is shown in Table 1 for various values of reactive loading. Note that even for relatively low system loadings, the reactive power margin is estimated with an error of just a few percent. It should be stressed that the reactive power margin can be estimated just from normal and low voltage power flow solutions; there is no need to actually generate the $Q-\ln (V)$ curve.

The reinterpretation of the energy measure as an area as described above required that one take the derivative of the energy margin with respect to a load parameter, and integrate once again with respect to this same load parameter. With this observation, it is equally valid to use active power load as the load parameter of interest. Consider the result when the active power load, $P_{L}$ is used. Here (4) would be replaced by:

$$
\frac{d \vartheta}{d P_{L}}=\frac{\partial \vartheta}{\partial P_{L}}+\frac{\partial \vartheta}{\partial x^{u}} \frac{\partial x^{u}}{\partial P_{L}}-\frac{\partial \vartheta}{\partial x^{s}} \frac{\partial x^{s}}{\partial P_{L}}
$$

with

$$
\frac{\partial \vartheta}{\partial \mathrm{P}_{\mathrm{L}}}=\boldsymbol{\alpha}^{\mathrm{u}}-\boldsymbol{\alpha}^{\mathbf{s}}
$$

As per observation (iv) above, the partial derivative terms $\partial \vartheta / \partial \mathrm{x}^{\mathrm{u}}$ and $\partial \vartheta / \partial x^{s}$ will again be identically zero along paths defined by the equilibrium power flow solutions $x^{\mathrm{u}}\left(P_{L}\right)$ and $x^{\mathrm{s}}\left(P_{L}\right)$. Similarly, as was the case when the system reached a reactive loading limit, one will have an active power loading limit at which $\vartheta\left(x^{u}\left(P_{L}^{\max }\right), x^{8}\left(P_{L}^{\max }\right)\right)=0$. One therefore obtains a result analogous to (5), with 


$$
\theta\left(x^{u}\left(P_{L}^{0}\right), x s\left(P_{L}^{0}\right)\right)=\int_{P_{L}^{0}}^{P_{L}^{\max }}\left(\alpha u\left(P_{L}\right)-\alpha s\left(P_{L}\right)\right) d P_{L} .
$$

It is important to note that so long as $P_{\mathrm{L}}^{0}$ and $Q_{L}^{0}$ are associated with the same operating point, then $\vartheta\left(x^{\mathrm{u}}\left(\mathrm{P}_{\mathrm{L}}^{0}\right), \mathrm{x}^{\mathrm{s}}\left(\mathrm{P}_{\mathrm{L}}^{0}\right)\right)=\vartheta\left(\mathrm{x}^{\mathrm{u}}\left(\mathrm{Q}_{\mathrm{L}}^{0}\right), \mathrm{x}^{\mathrm{s}}\left(\mathrm{Q}_{\mathrm{L}}^{0}\right)\right.$; i.e., the right hand sides of (5) and (7) are equal. While (5) and (7) give two very different forms of integral expression, with very different curves (P- $\alpha$ versus $Q-\ln (V))$, they must enclose the same area. Indeed, in the numerical examples to be examined in the following section, it will in some cases prove more convenient to use the $P-\alpha$ area in calculations. Also note that the P- $\alpha$ curve produces an area closely related to the decelerating area that would be calculated in a classic equal area criterion, except that the model used here includes reactive power balance constraints that are not considered in simple transient stability/equal area studies. Therefore the unstable equilibrium of interest, $x^{\mathrm{u}}\left(\mathrm{P}_{\mathrm{L}}^{0}\right)$, will not be related to that used in the equal area criterion.

Figure 3 plots the $P-\alpha$ curve for the same initial operating point as in Figure 2. Since both figures correspond to the same initial operating point, the two areas are identical, being equal to the energy measure of 0.861 . The area enclosed by this curve remains approximately parabolic, so the real power margin (defined as $\mathrm{P}_{\mathrm{L}}^{\max }$ minus the base case real power load) can be estimated as:

$$
\mathrm{P}_{\mathbf{L}}^{\max }=\frac{3}{2} \frac{\text { energy measure }}{\alpha^{*} \text { MVA base }} .
$$

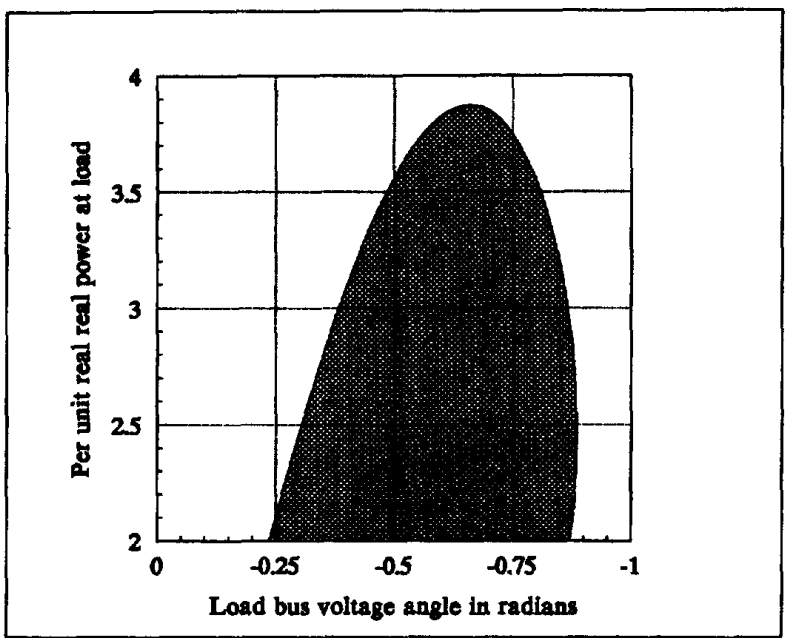

FIGURE 3: P- $\alpha$ Curve for Same Example as that of Figure 2

For this example, a maximum of 187.3 MW of constant $P$ characteristic load can be added before the critical level is reached. Table 2 compares the estimated to the actual real power margins. Again quite reasonable approximations can be made even when the system is quite far from voltage collapse, and the accuracy of the approximation improves as the system loading is increased.

A related and perhaps more intuitive relationship between the energy measure and the area enclosed by a P- $\alpha$, curve at a load bus can also be developed by reconsidering (2). The P- $\alpha$ curve for the two bus system is obtained by changing the real load power $P$ and holding the reactive load $Q$ constant. As the $P-\alpha$ curve is traced, the state $X=$ $(\alpha, V)$ will change along a path in state space from the high voltage solution $x^{\mathbf{s}}$ to the low voltage solution $x^{u}$. The energy measure can be evaluated by integrating along this path using formula (2) (the integration yielding the energy measure is path independent). In the integration along this path, the real power mismatch $f(x)$ at the load is nonzero and the reactive power mismatch is zero so that the integration reduces to the integration of the real power mismatch with respect to the bus angle:

$$
\vartheta\left(x^{u}, x^{z}\right)=\int_{x^{z}=\left(\alpha^{z}, v^{z}\right)}^{x^{u}=\left(\alpha^{u}, v^{u}\right)}[f(\alpha, v), g(\alpha, v)]\left[\begin{array}{l}
d \alpha \\
d V
\end{array}\right]=\int_{\alpha^{z}}^{\alpha^{u}} f(\alpha, v) d \alpha
$$

Moreover, if we regard the real load power $P$ as varying as a function of $\alpha$ and write $P^{0}$ for the base case real load power at $x^{3}$, then the real power mismatch $f(\alpha, v)=P-P^{0}$ and

$$
\vartheta\left(x^{\mathrm{u}}, \mathrm{x}^{\mathrm{s}}\right)=\int_{\alpha^{\mathrm{s}}}^{\alpha^{\mathrm{u}}}\left(\mathrm{P}-\mathrm{P}^{0}\right) d \alpha
$$

which is clearly the area enclosed by the $P-\alpha$ curve and the line $P=P^{0}$.

This argument extends easily to a P- $\alpha$ curve for a system with many buses. The integration path is obtained by changing the real load power at a given bus. Along this integration path, all other real and reactive load power mismatches are zero, so that the integration reduces to the integration of the real power mismatch at the given bus in a manner similar to equation (8). The rest of the argument is unchanged.

A related argument establishes that the energy measure for the two bus system is the area enclosed by the Q-V curve if the voltage is given a logarithmic scale. Evaluate the energy measure integral (2) along the path given by changing the reactive load power and holding the real power constant. Then

$$
\vartheta\left(x^{u}, x^{s}\right)=\int_{x^{8}=\left(\alpha^{s}, v^{s}\right)}^{x^{u}=\left(\alpha^{u}, v^{u}\right)}[f(\alpha, V), g(\alpha, v)]\left[\begin{array}{l}
d \alpha \\
d V
\end{array}\right]=\int_{v^{s}}^{v^{u}} g(\alpha, V) d V .
$$

If we regard the reactive load power $Q$ as varying as a function of $V$, and write $Q^{\circ}$ for the base case reactive demand, then $g(\alpha, V)=$ $\tilde{\mathbf{g}}(\alpha, V) / \mathrm{V}=\left(\mathrm{Q}-\mathrm{Q}^{\circ}\right) / \mathrm{V}$ and

\begin{tabular}{|c|c|c|c|c|c|}
\hline \multirow[t]{2}{*}{$\begin{array}{l}\text { MW load } \\
\text { at bus } 2\end{array}$} & \multirow[t]{2}{*}{$\begin{array}{l}\text { Energy } \\
\text { measure }\end{array}$} & \multicolumn{2}{|c|}{$\begin{array}{l}\text { Bus } 2 \text { volbage } \\
\text { angle In radians }\end{array}$} & \multicolumn{2}{|c|}{$\begin{array}{l}\text { Roal power } \\
\text { margin (MW) }\end{array}$} \\
\hline & & $\begin{array}{l}\text { Operable } \\
\text { solution }\end{array}$ & $\begin{array}{l}\text { Low } \\
\text { solution }\end{array}$ & Estimated & Actual \\
\hline 200 & 0.8609 & -0.236 & -0.871 & 203.3 & 187.3 \\
\hline 250 & 0.5548 & -0.304 & -0.886 & 143.1 & 137.3 \\
\hline 300 & 0.2867 & -0.383 & -0.886 & 89.0 & 87.3 \\
\hline 350 & 0.0812 & -0.484 & -0.809 & 37.5 & 37.3 \\
\hline 387 & 0.0001 & -0.643 & -0.675 & 0.3 & 0.3 \\
\hline
\end{tabular}

$$
\vartheta\left(x^{\mathbf{u}}, x^{s}\right)=\int_{v^{s}}^{v^{u}} \frac{Q-Q^{0}}{V} d V=\int_{\ln V^{s}}^{\ln V^{u}}\left(Q-Q^{0}\right) d(\ln V)
$$

which is clearly the area enclosed by the Q-lnV curve and the line $Q=Q^{\circ}$. This argument also extends easily to the $Q-\ln V$ curve for a multiple bus system.

TABLE 2 : Accuracy of estimated real power margin

In addition io providing estimates of the maximum real and reactive power which can be supplied to the load bus, the energy measure can also be used to provide an estimate of the maximum additional MVA that can be supplied at a particular power factor; this limit will be denoted as $S_{\max }$ (pf). This also corresponds to choice of a particular integration path. Here the path is chosen so that the real and reactive mismatches maintain a constant relative power factor. By again assuming a parabolic area, the value of $S_{\max }(\mathrm{p} f)$ can be estimated as: 


$$
S_{\max }=\frac{3}{2} \frac{\text { energy measure } * \text { MVA base }}{\mathrm{pf}^{*}\left(\alpha^{s}-\alpha^{\mathrm{v}}\right)+\sqrt{1-(\mathrm{pf})^{2} *\left(\ln \left(V^{v}\right)-\ln \left(V^{y}\right)\right)}} .
$$

Figure 4 compares the estimated to acmal value of $S_{\max }$ for the two bus system with a power factor of 0.894 (P/Q ratio of 2.0). For extremely low levels of loading the estimated value is quite conservative. This is because the curves' areas are no longer well approximated as parabolic over this wide a range. However for high loads, where one is most concerned about accuracy, the agreement between the true value and the estimate is quite good.

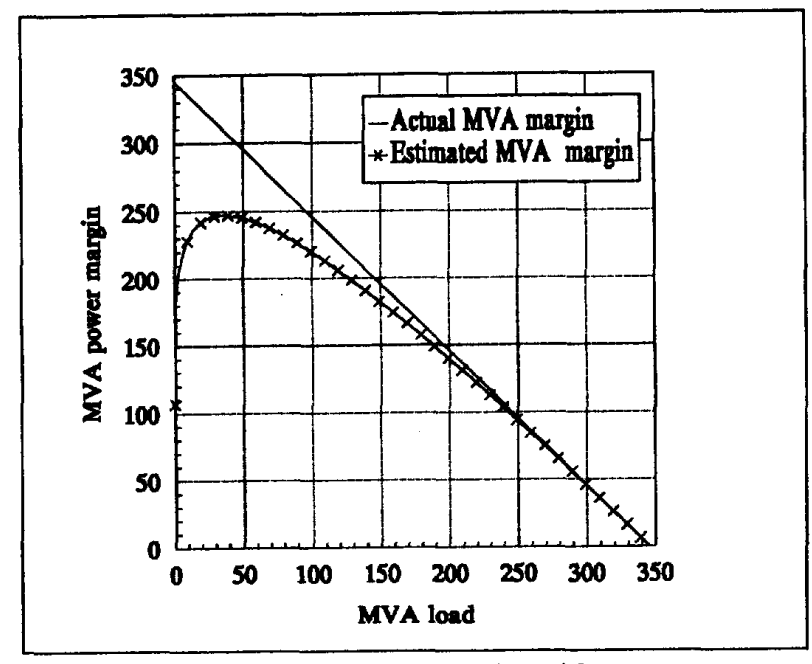

FIGURE 4: Actral vs. Estimated $S_{\max }$

The point to be emphasized in the preceding discussion is that the energy measure itself need only be determined once for a particular operation point, and that its determination does not require that any assumptions be made about future system parameter (e.g. load) variation. However, once the energy measure has been determined, a number of complementary estimates of system security follow for various postulated patterns of load increase. For example, at the loading of $200 \mathrm{MW}$ and $100 \mathrm{Mvar}$, the same energy measure of 0.861 was used to estimate the reactive power margin at $108.0 \mathrm{Mvar}$, the real power margin as 203.3 MW, and for an assumed load power factor of 0.894 , a margin of 121.9 MVA. Since the calculation of these security estimates is computationally trivial, as many as are necessary could be performed. If greater accuracy is required, a sequence of three or four energy margin calculations along the postulated path of load increase can be used to improve this estimate, as described in [8].

\section{APPLICATION TO MULTIPLE BUS SYSTEM}

The concepts from the previous section can be easily generalized to a systems of arbitrary size. Moreover, simple approximations allow an accounting for transmission losses in the energy evaluations. These extensions can be accomplished by first replacing the scalar equations (1a) and (1b') with the corresponding vector mismatch functions at every bus in the system (except the slack), using a sign convention for $P_{i}$ and $Q_{i}$ that is positive for injections:

$$
\begin{aligned}
& f_{i}(\alpha, V)=P_{i}-\sum_{j=1}^{n} B_{i j}\left|v_{i}\right|\left|v_{j}\right| \sin \left(\alpha_{i}-\alpha_{j}\right) \\
& -\sum_{j=1}^{n} G_{i j}\left|v_{i}^{s}\right|\left|v_{j}^{s}\right| \cos \left(\alpha_{i}^{s}-\alpha_{j}^{s}\right)
\end{aligned}
$$

$$
\begin{aligned}
& g_{i}(\alpha, V)=\left(V_{i}\right)^{-1}\left(Q_{i}+\sum_{j=1}^{n} B_{i j}\left|v_{i}\right|\left|v_{j}\right| \cos \left(\alpha_{i}-\alpha_{j}\right)\right) \\
& -\left(V_{i}^{s}\right)-1 \sum_{j=1}^{n} G_{i j}\left|v_{i}^{s}\right|\left|v_{j}^{s}\right| \sin \left(\alpha_{i}^{s}-\alpha_{j}^{s}\right) .
\end{aligned}
$$

The reader should note that the terms related to line conductance $G_{i j}$ involve only constant values of voltages and phase angles evaluated at the operating point. This approximate treatment of losses was introduced in [9]. With these terms, (9) and (10) yield zero mismatch at the operating point, but only approximate the true mismatch equations at all other points. Since the added terms are constants with respect to the variable of integration, the resultant energy measure is still exactly integrable (i.e. there is no path dependence in the integral).

With these changes, (2) becomes a vector integration in $2 n$ variables, where $n$ is the number of buses (other than the slack) in the system. This integration is still performed along a path between the current operating point and a particular low voltage solution. Somewhat surprisingly, the energy measure for a lossless system remains exactly equal to the area of the $Q-\ln (V)$ curve for a single bus $i$. The derivation remains identical to that of the single bus case in (4), with the key observation that even in the multiple bus system,

$$
\frac{\partial \vartheta}{\partial Q_{i}}=\ln \left(V_{i}^{s} / N_{i}^{y}\right) ;
$$

i.e., the integrand analogous to that in (5) remains a function only of voltage at bus $i$.

When losses are considered, the second term in the multiple bus analogy to (4) is no longer identically zero, since the gradient of the energy function at the low voltage solution yields the approximate mismatch equations. Therefore, for a system with losses, the energy measure approximates the area of the $Q-\ln (V)$ curve. Similar reasoning can be used to show that the energy measure approximates the area of the $P-\alpha$ curve for $a$ bus $i$. The effectiveness of this approximation will be explored in the examples to follow.

In a two bus system there is at most a single low voltage power flow solution. For the multiple bus system there can be a large number of separate power flow solutions (the maximum possible number grows exponentially with network size [11]). However, computational experience reported in [10] indicates that the set of desired low voltage solutions can typically be restricted to just the $\mathrm{n}$ solutions found using the rectangular Newton-Raphson power flow with a low initial voltage guess at a single bus. Computationally efficient methods to further restrict the number of solutions to be calculated are discussed in [12]. For a large system the energy measures found using the different low voltage solutions are associated with voltage security of a particular area in the system.

The use of energy methods to determine security measures for a larger system is demonstrated using the IEEE 118 bus system. In order to produce an example of voltage collapse for the system, loads were assumed to be a linear function of a parameter $k$ ( $k=1$ for basecase). To represent the change in generation necessary to track this load increase, reasonable generator participation factors were assumed. Generator reactive power limits were modeled using methods described in [9]. Figure 5 plots the variation in the energy measures with respect to total system load + losses for the six low voltage solutions with the lowest associated energy values. Thus at a particular operating point/loading level, each energy measure provides an indication of the voltage security in an area of the system centered about the indicated bus. Note that the energy measures are nearly linearly with respect to changes in the system operating point, and experience no discontinuities when generator reactive power limits are reached (in the Figure 5 example more than $\mathbf{3 0}$ generators reach their limits as the load increases). Thus Figure 5 illustrates the "direct" use of the energy 
336

measure to indicate proximity to voltage instability by repeated margin calculations over a range of loading levels.

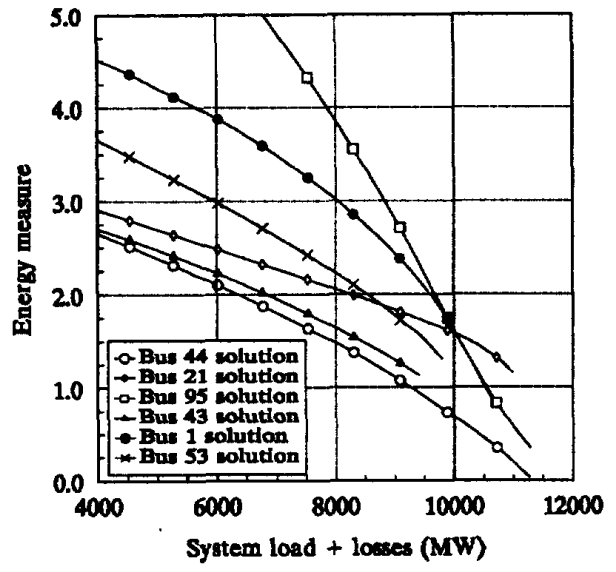

FIGURE 5: Energy Measure Variation for IEEE 118 Bus System

As an alternative to this direct use of the plot of the energy measure, the previous section has argued that energy margins can also be used to approximate more intuitive measures of system security. To illustrate in a multiple bus example, consider again the relation of the energy measure to the $P-\alpha$ or the $Q-\ln (V)$ curve area for the case were load is changing only at a single bus. Using the parabolic area approximation, estimates of the real, reactive and MVA power margins from any given operating point can be calculated. Table 3 shows these values for the case of bus 44 being loaded (note that this pattern of load increase is much different than that assumed in Figure 5; the margins are therefore much lower in Table 3). Each of these power margins is obtained from a single energy margin calculation. For comparison, the actual areas of the $Q-\ln (V)$ and $P-\alpha$ curves (computed by numerical integrating the curves) are also shown.

\begin{tabular}{|c|c|c|c|c|c|c|c|c|c|}
\hline \multirow[t]{2}{*}{$\begin{array}{l}\text { System } \\
\text { load }\end{array}$} & \multirow[t]{2}{*}{$\begin{array}{l}\text { Energy } \\
\text { measure } \\
\end{array}$} & \multirow[t]{2}{*}{$\begin{array}{l}\text { Area } \\
Q-\ln (V)\end{array}$} & \multicolumn{2}{|c|}{$\begin{array}{l}\text { Reactlve } \\
\text { margin (Mvar) }\end{array}$} & \multirow[t]{2}{*}{$\begin{array}{l}\text { Aroa } \\
\text { P- } \alpha\end{array}$} & \multicolumn{2}{|c|}{$\begin{array}{l}\text { Real power } \\
\text { margin (Why) }\end{array}$} & \multicolumn{2}{|c|}{$\begin{array}{l}\text { MVA margin } \\
\text { at } \mathrm{pl}=0.894\end{array}$} \\
\hline & & & True & Est. & & True & Est. & True & Est. \\
\hline 4000 & 2.65 & 2.85 & 167 & 114 & 1.97 & 237 & 420 & 183 & 165 \\
\hline 6000 & 2.11 & 2.31 & 144 & 108 & 1.57 & 201 & 330 & 154 & 147 \\
\hline 8000 & 1.49 & 1.64 & 117 & 86 & 1.11 & 155 & 238 & 119 & 119 \\
\hline 10000 & 0.69 & 0.75 & 76 & 75 & 0.50 & 92 & 144 & 70 & 82 \\
\hline 11000 & 0.20 & 0.18 & 38 & 46 & 0.13 & 41 & 71 & 31 & 45 \\
\hline
\end{tabular}

TABLE 3 : Comparison of actual to estimated power margins for Bus 44

While the estimated margins are not as accurate as for the two bus system, they do demonstrate that the energy measures can provide quite reasonable estimates of system security, with the margins decreasing to zero at the load level associated with voltage collapse. Similar estimates of real, reactive and MVA power margins can be computed at other buses by using the loading pattern associated with the given bus. For example at a system load of 10,000 MW the estimated MVA margins (assuming basecase power factor) at bus 1 is 100 compared to an actual of 93 MVA, at bus 21 it is 119 MVA compared to 126 MVA and at bus 95 it is 212 MVA compared to an actual of $180 \mathrm{MVA}$. We envision that these types of margins will provide a very low cost screening tool to identify patterns of load increase that produce threats to security. Once the threatening patterns of load increase are identified, more accurate power margins can be obtained from energy calculations analogous to those for Figure 5 , or from alternate means of computing load power margins such as [13], [14].

In addition to providing estimates of the power margins at a single bus, the energy measure can also be employed to provide more system wide security measures. This can be done by again choosing a particular integration path for the integral expression in (2). Rather than following a path that produces nonzero mismatch only at a single bus, the path is chosen so that the load/generation is changing at some or all of the system buses based upon assumed participation factors. For example, by fixing coefficients $\beta_{\mathrm{P}, 1}$ and $\beta_{Q, i}$ at each bus, and letting $\Delta P$ be the total added system load, each bus load follows a linear path defined by:

$$
\begin{aligned}
& P_{i}=P_{i}^{\text {base }}+\beta_{P, i} \Delta P, \\
& Q_{i}=Q_{i}^{\text {base }}+\beta_{Q, i} \Delta P .
\end{aligned}
$$

With the appropriate shape assumed for the $Q-\ln (V)$ and $P-\alpha$ curves, an estimate of the maximum allowable increase in system load, $\Delta P^{\mathrm{max}}$, is determined as:

$$
\begin{aligned}
& \Delta P_{\max }= \\
& \sum_{i=1}^{n} \beta_{P, i} \gamma_{P, i}\left|\alpha_{i}^{8}-\alpha_{i}^{u}\right|+\beta_{Q, i} \gamma_{Q, i}\left|\ln \left(V_{i}^{s}\right)-\ln \left(V_{i}^{u}\right)\right|
\end{aligned}
$$

where $\gamma_{P, 1}$ and $\gamma_{Q, j}$ are scalars that depend upon the assumed geometry of the real and reactive curves; variations on the parabolic shape assumption will be outlined below.

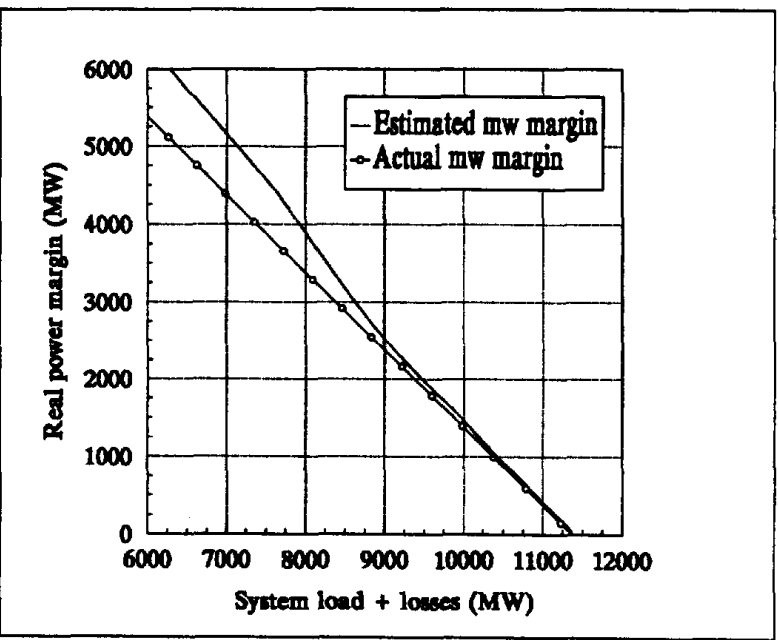

FIGURE 6: Comparison of Estimated to Actual Values of $\Delta \mathrm{P}_{\max }^{\max }$

As an example, Figure 6 plots the estimated and actual values of $\Delta P^{\max }$ as a function of system load for the same pattern of load increase used in Figure 5. In calculating the estimated value of $\Delta \mathrm{P}^{\mathrm{max}}$, the assumption was made that the loads and generation are a linear function of a parameter $k$, and that the $P-\alpha$ curve shapes were parabolic at all buses $\left(\gamma_{P, i}=2 / 3\right)$. The $Q-\ln (V)$ curve shapes were assumed parabolic at the low voltage bus and rectangular elsewhere. Intuitively this assumption arises because most of the voltage deviation occurs at the low voltage bus, while the voltages at other buses in the low voltage solution tend to be nearly constant because of voltage regulation at nearby generating buses; $Q-\ln (V)$ curves resulting from nearly constant voltages were better approximated by a rectangular shape.

Different scenarios can be studied using (11) with changes in the assumed participation factors; the energy measure need only be determined once. For example, from a base case loading of 10000 
MW, the same energy measure of 0.69 for the bus 44 rogion was used both to determine the power margins shown in Table 4, and the value of $\Delta \mathrm{P}^{\mathrm{mxx}}$ from Figure 6. Consider an alternate load increase scenario in which load participation is limited to just bus 44 and its first neighbor buses of 43 and 45 . A new value of $\Delta P \max$ for this scenario is computed from (11) using the same energy value in the numerator, but changing the participation factors in the denominator so that the only nonzero values correspond to these three buses. In this case, from a base loading of $10000 \mathrm{MW}$, the calculated value of $\Delta \mathrm{Pmax}^{\mathrm{ma}}$ $97 \mathrm{MW}$, compared to an actual of $92 \mathrm{MW}$. For a study scenario that extends the group of buses with increasing load to bus 44 with its first and second neighbors, the calculated value of $\Delta \mathrm{P}^{\mathrm{max}}$ is $208 \mathrm{MW}$, vs. an actual value of $201 \mathrm{MW}$.

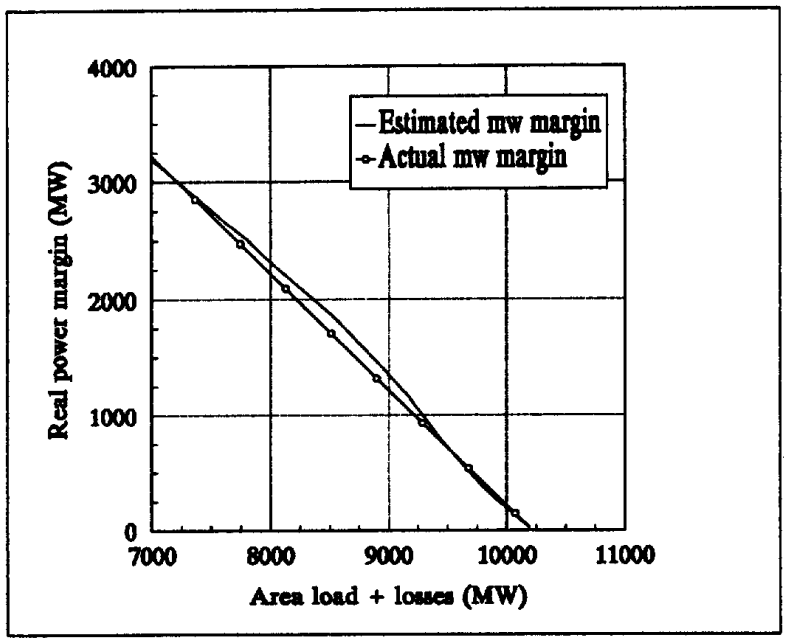

FIGURE 7 : Comparison of Estimated versus Actual Value of $\Delta \mathrm{P}^{\max }$ for $\mathbf{4 1 5}$ Bus Test System

As a final example the method was tested on the 415 bus system from [12]. The voltage collapse scenario studied here increased load uniformly at all buses in a contiguous 203 bus area within the network; generation was then varied to maintain constant interchange between this area and the rest of the system. For an increasing sequence load levels, a value of $\Delta P^{\max }$ was again estimated via (11). Using the same rules for determining $\gamma_{P, i}$ and $\gamma_{Q, j}$ as for case described in Figure 6 , Figure 7 plots the estimated and actual values of $\triangle \mathrm{P}^{\mathrm{max}}$ as a function of area load. Again, very accurate estimates of $\Delta \mathrm{P}^{\mathrm{max}}$ are obtained even when the system is far from the critical loading limit.

\section{v. CONCLUSIONS}

This paper has shown that for system models with constant $P-Q$ loads, the energy based voltage security measure can be related to area enclosed by a Q-InV curve, or that of a P- $\alpha$ curve. While the energy measure itself is applicable to more general voltage dependent load models, this paper has argued that relationship in the simple $P-Q$ load case is useful for two reasons. First, it provides an intuitive interpretation of the energy measure that should make its application more acceptable in an operational environment. In particular, the interpretation as an area shows that the energy measure captures information about both the distance from the operating point to the low voltage power flow solution, and the load power margin. Moreover, a single energy measure computation can be used to approximate loadability margins for a whole family of load increase patterns starting from the base case at which the energy is computed. The computational cost of these loadability margin approximations is extremely low, providing an efficient screening tool to identify patterns of load increase most threatening to the system's voltage security. Once a managable number of loading patterns are identified for further study, more accurate estimates of the loadability margins of interest can be obtained by repeated energy calculations, or by alternate methods in the literature. The application of these ideas was illustrated with sample computations in a 118 bus test system, and a 415 bas test system.

\section{ACKNOWLEDGMENTS}

The authors gratefully acknowledge support by the National Science Foundation under grants ECS-8857019 and ECS-9157192.

\section{REFERENCES}

[1] Voltage Stability of Power Systems: Concepts, Analytical Tools, and Industry Experience, IEEE 90 TH0359-2-PWR.

[2] Procoedings: Bulk Power System Voltage Phenomena - Voltage Stability and Security, EPRI Report EL-6183, Jan. 1989.

[3] Procoedings: Bulk Power System Phenomena - 1991: Voltage Stability and Security, McHenry, MD, Aug. 1991.

[4] I. Dobson and H.-D. Chiang, "Towards a Theory of Voltage Collapse in Electric Power Systems," Systems and Control Letters, vol. 13, pp. 253-262, 1989.

[5] E. H. Abed and P. P. Varaiya, "Nonlinear Oscillations in Power Systems," Electric Power Energy Sys., vol. 6, pp. 37-43, Jan. 1984.

[6] Y. Mansour, "Voltage Stability Limit: B. C. Hydro's practice," pp. 2.9-2.25, from [2].

[7] M. B. Awad, H. M. Zein Ei-Din and C. E. Graham, "Preventative Measures to Voltage Collapse in Bulk Electricity Systems," pp. 2.27-2.45, from [2].

[8] C. L. DeMarco and T. J. Overbye, "An Energy Based Security measure for Assessing Vulnerability to Voltage Collapse," IEEE Trans. on Power Sys., vol. 5, no. 2, May 1990.

[9] T. J. Overbye and C. L. DeMarco, "Voltage Security Enhancement Using Energy based Sensitivities," IEEE Trans. on Power Sys., vol. 6, no. 3, Aug. 1991.

[10] T. J. Overbye and C. L. DeMarco, "Improved Techniques for Power System Voltage Stability Assessment Using Energy Methods," IEEE Trans. on Power Sys., vol. 6, no. 4, Nov. 1991.

[11] F. M. A. Salam et al., "Parallel Processing for the Load Flow of Power Systems: the Approach and Applications," Proc. of the 28th IEEE Conf. on Decision and Control, pp.2173-2178, Tampa, FL, Dec. 1989.

[12] T. J. Overbye, "Use of Energy Methods for On-Line Assessment of Power System Voltage Security," IEEE PES 1992 Winter Power Meeting, paper \# 92 WM 121-4 PWRS, January 1992.

[13] T. Van Cutsem, "A Method to Compute Reactive Power Margins with Respect to Voltage Collapse," IEEE Trans. on Power Sys., vol. 6, no. 2, pp.145-156, Feb. 1991.

[14] C. A. Caxizizares et al, "Point of Collapse Methods Applied to AC/DC Power Systems," IEEE Trans. on Power Sys., vol. 7, no. 2, pp.673-683, May 1992.

\section{BIOGRAPHIES}

Thomas J. Overbye received his $\mathrm{Ph}$. D. from the University of Wisconsin-Madison in 1991, and is an Assistant Professor with the E.C.E. Department of the University of Illinois at Urbana-Champaign. Ian Dobson received his $\mathrm{Ph}$. D. from Cornell University in 1989, and is an Assistant Professor with the E.C.E. Department of the University of Wisconsin-Madison.

Christopher L. DeMarco received his Ph. D. from the University of Califomia, Berkeley in 1985, and is an Associate Professor with the E.C.E. Department of the University of Wisconsin-Madison. 


\section{Discussion}

M. K. Pal: The authors have presented an interesting approach to determining voltage stability margin. We would, however, like to point out that the voltage instability point as stated in the paper is actually a loading limit as determined by network laws. For constant MVA load as considered in the paper, the loading limit will correspond to voltage stability limit only when the load dynamic response speed is slow compared to the speed of response of system voltage control. For fast response loads, actual voltage stability limit can be well below the limit defined by the authors.

If the load characteristic is such that the voltage stability limit and the maximum loading point are the same, the authors' method would seem to be effective in the two-bus system discussed in Section III. Although the authors do not clearly state how the energy measure would be computed, presumably it will come from equation (2). The reactive power margin would then be calculated using the expression given in the paper.

We have, however, strong reservation about the applicability of the methodology in large systems. While methods are available for reliable computations of the low-voltage solution points, selection of the appropriate low-voltage solution can be tricky. In a large network, selecting one bus for load increase while maintaining loads at other buses at the initial level will certainly give an unrealistic limit. Generally, when the system loading increases, loadings at most of the load buses would change by varying amounts. If the load is allowed to increase at all the buses according to some participation factor, then the choice of the lowvoltage solution point, which in turn will determine the shape of the $\mathrm{Q}-\ln V$ (or $\mathrm{P}-\alpha$ ) curves at the various buses, will be open to question, and so will the reliability of the methodology. The results will also depend to a large extent on the assumed shape of the Q$\ln \mathrm{V}$ and $\mathrm{P}-\alpha$ curves.

It is not clear how the energy measure integral (2) would be evaluated in a multiple bus system. Too many variables, not independent of each other, are involved. However, an expression for the energy measure can be written by analogy with that for the two-bus system, so that its gradients (iv) are the power balance equations, and (5) is satisfied. (Note that, in a multiple bus system, it does not follow from (2) that these gradients yield the power balance equations.) It would be useful if the authors would show a few steps in the evaluation of the integral (2) in a multiple bus system, and the resulting expression of the energy measure.

The authors note that the energy measure does not experience discontinuity when generator reactive power limits are encountered. This might be due to the specific generator modeling at reactive limit used by the authors. When a generator hits reactive limit, the appropriate modeling is by its internal voltage behind synchronous reactance, not by simply changing from a P-V bus to a P-Q bus. With appropriate modeling, it is clear from (2) that there will be a discontinuity every time a generator hits reactive limit.

It is not clear how a single energy measure calculated at a given operating point can provide reactive margins at other reactive loadings, since Mvar loading does not change independently of MW loading.

R. M. Hassan and C. O. Nwankpa (ECE Department, Drexel University, Philadelphia, PA):

The authors should be commended for their interesting paper on providing physical interpretation of the energy based voltage security measure (using $Q-\ln V$ and $P-\alpha$ curves) previously developed by them. In addition, an application of developing approximated loadability margins has been developed. We agree with the authors that "The computational cost of these loadability margin approximations is extremely low..." hence providing an impetus for its application. the discussors will appreciate authors' comments on the following.

In practice familiar Q-V curves provide information aimed at obtaining so-called var margins to voltage collapse. Energy based security indices have provided another route to achieving this result. In both cases, voltage security is investigated in terms of increasing reactive load. These studies falls under the category of small disturbance stability analysis, hence the justification of the comparison performed in the paper. Have the authors looked at implementing the energy based voltage security index in terms capturing voltage vulnerability profiles of systems due to large disturbances? If so, can the authors provide insight into a proper comparison that may be performed in the same light as in this paper? In other words, is the energy based security measure an appropriate substitute for a time domain simulation approach to capturing trajectories of voltage collapse.

Manuscript received March 1, 1993.

T.J. Overbye, I. Dobson and C.L DeMarco: We would first of all like to thank the discussers for their interest in our paper.

Concerning M.K. Pal's discussion, we would agree that, as mentioned in the paper, voltage collapse is here assumed to be the dynamic consequence of a saddle node bifurcation in which the system's operating equilibrium disappears, and can therefore be considered a loading limit. As stated in the paper's introduction, the assumed scenario of our analysis is one in which the system state is moving from a point of relative security to one of increased vulnerability to voltage instability through a quasi-static variation (time scale tens of minutes to hours). The system representation and modeling used is intended to capture system behavior during this relatively slow evolution, and to identify the critical loading. Our model is not intended to accurately predict system behavior in the final moments of collapse, when the operating point has lost stability and the system state is diverging with (typically) rapidly declining voltages. Also, we have not considered oscillatory instabilities caused by Hopf bifurcations, and would agree with the discusser that for certain dynamic models (which could certainly include fast response loads) the actual voltage stability limit could be below the limits given in the paper. However, we would argue that for a large class of plausible system dynamic models, the measures proposed in the paper do provide a useful assessment of the system's voltage stability. For example, it is known that adding certain general types of dynamics to a load power balance equation does not alter the saddle node bifurcation voltage stability limit [C2]. Furthermore, the application of such Q-V and P-V based measures is well established in utility practice.

The discusser raises the question of whether an expression of the form (2) can be used to obtain an energy measure for a multiple bus system. We want to stress that such a closed form energy expression is easily expressible in closed form, as shown below in (c1), and was used throughout the paper's calculations. The explanation of the measure focused on the two bus example for simplicity only, given that the derivation for the multiple bus 
case has been addressed in many previous works (see, for example [C1], and our papers [8], [9], [10]). In the two bus example of the paper, the right-hand side of (2) (last two lines in the equation) provide the closed form expression for the energy measure in terms of the high and low voltage power flow solutions. For example consider the two bus system with per unit values of $B_{12}=$ $-\mathrm{B}_{22}=10, \mathrm{P}_{\mathrm{L}}=2.0, \mathrm{Q}_{\mathrm{L}}=1.0,\left(\alpha^{\mathrm{s}}, \mathrm{V}^{\mathrm{s}}\right)=(-0.2360 \mathrm{rad}, 0.8554)$ and $\left(\alpha^{\mathrm{u}}, \mathrm{V}^{\mathrm{u}}\right)=(-0.8711 \mathrm{rad}, 0.2614) ;$ straightforward variable substitution in (2) gives the energy measure reported for Figure 2 of 0.861. The same type of expression and evaluation applies in the multiple bus case. As requested by the discusser, the closed form expression for the multiple bus energy measure used in the paper is given by:

$$
\begin{aligned}
& \vartheta\left(x^{s}, x^{u}\right)=-\frac{1}{2} \sum \sum^{n} B_{i j}\left|v_{i}^{u}\right|\left|v_{j}^{u}\right| \cos \left(\alpha_{i}^{u}-\alpha_{j}^{u}\right) \\
& \mathrm{i}=1 \mathrm{j}=1 \\
& \text { n } \mathbf{n} \\
& +\frac{1}{2} \sum \sum B_{i j}\left|v_{i}^{s}\right|\left|v_{j}^{s}\right| \cos \left(\alpha_{i}^{s}-\alpha_{j}^{s}\right) \\
& \mathrm{i}=\mathbf{1} \mathbf{j}=\mathbf{1} \\
& \mathrm{n} \mathrm{v}_{\mathrm{i}}^{\mathrm{u}} \\
& -\sum \int \frac{\mathrm{Q}_{i}(\mathrm{x})}{\mathrm{x}} \mathrm{dx}-\mathbf{P}^{\mathrm{T}}\left(\boldsymbol{\alpha}^{\mathrm{u}}-\boldsymbol{\alpha}^{\mathrm{S}}\right) \\
& \mathrm{i}=1 \mathrm{v}_{\mathrm{i}}^{\mathrm{s}} \\
& -\sum^{n}\left(\sum^{n}\left(G_{i j}\left|v_{i}^{s}\right|\left|v_{j}^{s}\right| \cos \left(\alpha_{i}^{s}-\alpha_{j}^{s}\right)\right)\left(\alpha_{i}^{u}-\alpha_{i}^{s}\right)\right) \\
& \mathrm{i}=1 \mathrm{j}=1 \\
& -\sum_{i=1}^{n}\left(\left(v_{i}^{s_{s}}\right)^{-1} \sum_{j=1}^{n}\left(G_{i j}\left|v_{i}^{s}\right|\left|v_{j}^{s}\right| \sin \left(\alpha_{i}^{s}-\alpha_{j}^{s}\right)\right)\left(v_{i}^{u}-v_{i}^{s}\right)\right) \text {. }
\end{aligned}
$$

It is important to note that each of the terms involving $Q_{i}(x)$ are integrals of a scalar function (reactive demand at bus $i$ ) of a scalar variable (voltage magnitude at that bus). Hence there is no issue of path dependence in these expressions, and the integral is easily evaluated in closed form when the reactive load $Q_{i}$ is an arbitrary polynomial or exponential function of bus voltage. As was the case for the two bus system, once the high and low voltage power flow solutions ( $\mathbf{x}^{\mathrm{s}}, \mathbf{x}^{\mathrm{u}}$ respectively) are known, numerical evaluation of the energy measure requires only straightforward substitution into (c1). The computational complexity is on the order of the calculation of the power flow mismatch vector. For the lossless case, simple partial derivative calculations confirm that the gradient of (c1) is indeed the vector of the power balance equations (with the reactive equation at bus $i$ scaled by $\left.\left(V_{i}\right)^{-1}\right)$. For the case with transmission line losses, the gradient yields a vector function that is exactly equal to the power mismatch equations only when evaluated at $\mathbf{x}^{\mathbf{s}}$; at all other points it only approximates the power balance equations.

M.K. Pal raises an interesting issue concerning the selection of the appropriate low voltage solution. For a set of load participation factors, the correct low voltage solution to use in the power margin calculation is the one which will ultimately coalesce with the high voltage solution if the load increase were allowed to continue (with the specified participation) to the point of bifurcation. The appropriate low voltage solution is therefore dependent upon the assumed participation factors. For cases in which the load increase is limited to a single bus (such as in the classic Q-V curve) the selection of the low voltage solution is almost invariably that found with an initial low voltage guess at the bus in question. We would agree that when the load is allowed to increase at a number of different buses this choice is somewhat more complicated. Based upon numerical experimentation we have found that almost without fail the correct low voitage solution to use is the one which yields the lowest power margin; this method was used for all examples in the paper. For realistic load participation factors these solutions tend to be the ones with the lowest associated energy measures. Computationally efficient methods for locating this set of low energy solutions are discussed in [10] and [13].

We would also agree that the exact power margins are dependent upon the precise shape of the $Q-\ln (V)$ and $P$-alpha curves. Therefore, the quality of our power margin estimates are dependent on the accuracy of the simple analytic expressions used to approximate these curves. As mentioned in the paper, theoretical considerations related to the saddle node bifurcation indicate this shape is asymptotically parabolic near the critical points. For operating points more distant from the critical point, numeric testing indicates that the assumed shapes proposed in the paper appear to work well on realistic systems (such as the 118 and 415 bus cases). Since the computational cost of these margins is quite low, they can be used to provide an efficient screening tool to identify patterns of load increase most threatening to the system's voltage security. As mentioned in the conclusion, if more accurate estimates of these loadability margins are required they can be obtained by repeated energy calculations, or by alternate methods.

Concerning M.K. Pal's comments on generator models, we note that the appropriate generator model depends in part upon the time scale of the problem. For the time scale considered in this paper (minutes to hours), the reactive output of a generator will be limited primarily by the values from its reactive capability curve. Therefore a var limited generator can be modeled accurately as a PQ bus as the system operating point moves quasi-statically towards the critical loading level. As noted earlier in this closure, we would not advocate these models to predict behavior beyond the critical loading level, as one might wish to do in a full time domain simulation of a voltage collapse incident. More detailed dynamic models of exciter saturation might be useful to capture exact voltage trajectories as the state diverges from the former operating point; it is our opinion that such detail is generally not needed to predict the critical point.

To address the question of continuity of the energy measure as generators encounter reactive limits, we would repeat the paper's assertion that the measure is indeed continuous in such cases. This may be easily understood via the integral term in $Q_{i}(V)$ in the definition of the energy measure (c1). Recall from the paper that for generators, $Q_{i}(V)$ represents a saturating function of generator var output. The var output $Q_{i}(V)$ for a generator will have a discontinuity in its slope at the point when the limit is reached. However, $Q_{i}(V)$ itself remains a continuous, though not smooth, function. The energy measure depends on the integral of $Q_{i}(x) / x$ with respect to $\mathrm{V}$, and is therefore guaranteed to be continuous over the always positive range of voltage magnitudes.

In regards to the discusser's last comment, we would like to thank him for giving us an opportunity to clarify this point. An energy measure, calculated for a given operating point, is used to approximate the power margins only at that operating point. Since the power margins are dependent upon the assumed load participation, the energy measure could be used to provide margins for a whole family of assumed load increase patterns. However, each of these margins is defined with respect to the original operating point. Thus, for the example in the paragraph immediately preceding Figure 7, the same energy value of 0.69 (calculated for the operating point of $10000 \mathrm{MW}$ load) is used to determine the MW, Mvar and MVA margins shown in the 10000 MW load row of Table 3, and the real power margin shown in 
Figure 6 corresponding to a load of $10000 \mathrm{MW}$. Other margins in the table and figure, associated with other loads, are determined using the energy measures associated with those loads.

In response to R.M. Hassan and C.O. Nwankpa, we are thankful for their interest in, and positive assessment of our method. Our emphasis to date has been in using energy methods to provide margins for the maximum loadability of a system. In a sense, this application can be considered a nonlinear small disturbance stability measure, in the sense that large structural changes or disturbances are not of direct interest in these studies. Energy based methods have, of course, been used to aid in the assessment of system transient stability. Therefore we believe that energy methods offer the potential for assessing system voltage stability following large disturbances. Hówever we have not as yet pursued a intensive research in that area. Preliminary results in this direction may be found in [C3], [C4]
[C1] M.A. Pai, Energy Function Analysis for Power System Stability, Kluwer Academic Publishers, Boston, 1989.

[C2] I. Dobson, "Observations on the geometry of saddle node bifurcation and voltage collapse in electric power systems," IEEE Transactions on Circuits and Systems, Part 1: Fundamental Theory and Applications, vol. 39, no. 3, March 1992, pp. 240-243.

[C3] D.J. Hill and I.A. Hiskens, "Dynamic analysis of voltage collapse in power systems," Proc. 31th IEEE Conf. Decision and Control, Tuscon, AZ, pp. 2904-2909, Dec. 1992.

[C4] T.J. Overbye, M.A. Pai and P.W. Sauer, "Some aspects of the energy function approach to angle and voltage stability analysis in power systems," Proc. 31th IEEE Conf. Decision and Control, Tuscon, AZ, pp. 2941-2946, Dec. 1992.

Manuscript received April 1, 1993. 